\title{
SPONTANEOUS RUPTURE OF AMYLOID SPLEEN
}

\author{
${ }^{*}$ MR. SHWETAL DIGHE \\ United Kingdom \\ MR. A. ZAIDI \\ United Kingdom \\ MR. SAMI FARHAT \\ United Kingdom
}

Article received on:

$21 / 03 / 2007$

Accepted for publication: $15 / 05 / 2007$

\begin{abstract}
Primary systemic amyloidosis $(A L)$ is characterized by the overproduction of immunoglobulin light chain proteins by a monoclonal, terminally differentiated B-lymphocyte or plasma cell clone. The free immunoglobulin light chains are deposited in an abnormal conformation as amyloid in a variety of organs in the body (1). Splenic involvement is seen in $4-13 \%$ of cases, however amyloidosis causing spontaneous rupture is very rare with only 31 cases reported so far. We report a case of 60-year old, suffering from Primary AL amyloidosis and multiple myeloma involving the liver, spleen and GI tract, who suddenly collapsed and developed rapid abdominal distension. CT scan suggested rupture of the spleen. She underwent emergency Splenectomy and had an uneventful recovery. It has been suggested that the deposition of amyloid in the basement membrane of the vascular endothelium leads to increased gap between junctions and leading to vascular fragility and propensity to rupture. The amyloid can also lead to defective interaction with platelets and predispose to spontaneous haemorrhage. Conclusion: Thus in a case of occult haemorrhage with Amyloidosis, diagnosis of rupture of spleen should be considered and CT scan should be performed if the patient is haemodynamically stable. Splenectomy is the ideal treatment for ruptured spleen.
\end{abstract}

Key words: $\quad$ Amyloidosis, Spontaneous, Rupture, Spleen.

\section{INTRODUCTION}

Primary systemic amyloidosis $(\mathrm{AL})$ is characterized by the overproduction of immunoglobulin light chain proteins by a monoclonal, terminally differentiated B-lymphocyte or plasma cell clone. The free immunoglobulin light chains are deposited in an abnormal conformation as amyloid in a variety of organs in the body ${ }^{1}$. Amyloidosis is an uncommon disease, with an incidence of 8 per million per year².

Symptomatic liver involvement is seen in about $15 \%$ of cases, splenomegaly in $4-13 \%$ of cases and Gastro intestinal involvement in $8 \%$ of cases, of which $1 \%$ show symptoms such as heamatemesis or prolonged nausea and vomiting in association with weight loss ${ }^{4}$.

\section{CASE REPORT}

A 60-year-old woman initially presented to the Royal Marsden hospital, feeling unwell and on examination had hepatomegaly. Her Liver function tests (LFT) and Renal function tests were deranged. She subsequently had a liver biopsy that revealed Amyloidosis. Her $\lg G$ paraprotein levels were raised at $8.1 \mathrm{gm} / \mathrm{l}$ and her Bone marrow aspirate revealed $7 \%$ of plasma cells. Light chain 
studies showed raised serum kappa monoclonal light chains 172(N-8.1-33mg/l). Upper GI endoscopies performed for an episode of heamatemesis revealed $\mathrm{GI}$ Amyloidosis. Diagnosis of AL Amyloidosis and Myeloma with hepatorenal and $G I$ involvement was confirmed. She was treated by, Autologous Stem Cell Transplant with Granulocyte colony stimulating factor (G-CSF) and high dose Melphalan.

She again was admitted 4 months later with nausea and feeling generally unwell. She suddenly collapsed in the evening and developed rapid abdominal distension. She became hypotensive and her haemoglobin $(\mathrm{Hb})$ dropped from $9.8 \mathrm{gm} / \mathrm{dl}$ to $6.1 \mathrm{gm} / \mathrm{dl}$. She was transfused with 6 units of blood and 2 units of fresh frozen plasma (FFP) and was transferred for surgical treatment at the St Helier Hospital. On admission her pulse was 130 and blood pressure of 90/60. Her blood report showed; $\mathrm{Hb}$ $6.0 \mathrm{gm} / \mathrm{dl}$; WCC-17.2; Platelets 189; Creatinine -251 ; Urea-20.2 and Normal LFT. A CT Scan was performed as her condition was stable which showed rupture of the spleen. Urgent Laparotomy was performed which revealed 5 litres of blood in peritoneum, enlarged liver and a ruptured spleen. Splenectomy was performed. Postoperative course was uneventful and patient received vaccinations against pneumococci, meningococci and hemophilus. She was discharged on long-term benzyl penicillin. Histology confirmed the diagnosis of amyloid spleen with capsular tears which weighed $810 \mathrm{gms}$.

\section{DISCUSSION}

CT scan remains the investigation of choice in cases of suspected intra-abdominal bleeding if circumstances permit it. It does alter the management in significant number of patients if the site of bleeding is hepatic or vascular aneurysms as they can be treated by intervention techniques ${ }^{2}$.

Spleen is the most common organ to undergo spontaneous rupture due to its friable and vascular nature. A Literature search revealed 352 cases of rupture of spleen from 1966 till date with malaria and infectious mononucleosis as the most common aetiological factors. However the incidence of spontaneous rupture of spleen in case of Amyloidosis is very rare with only 21 cases documented so far ${ }^{2,5}$.

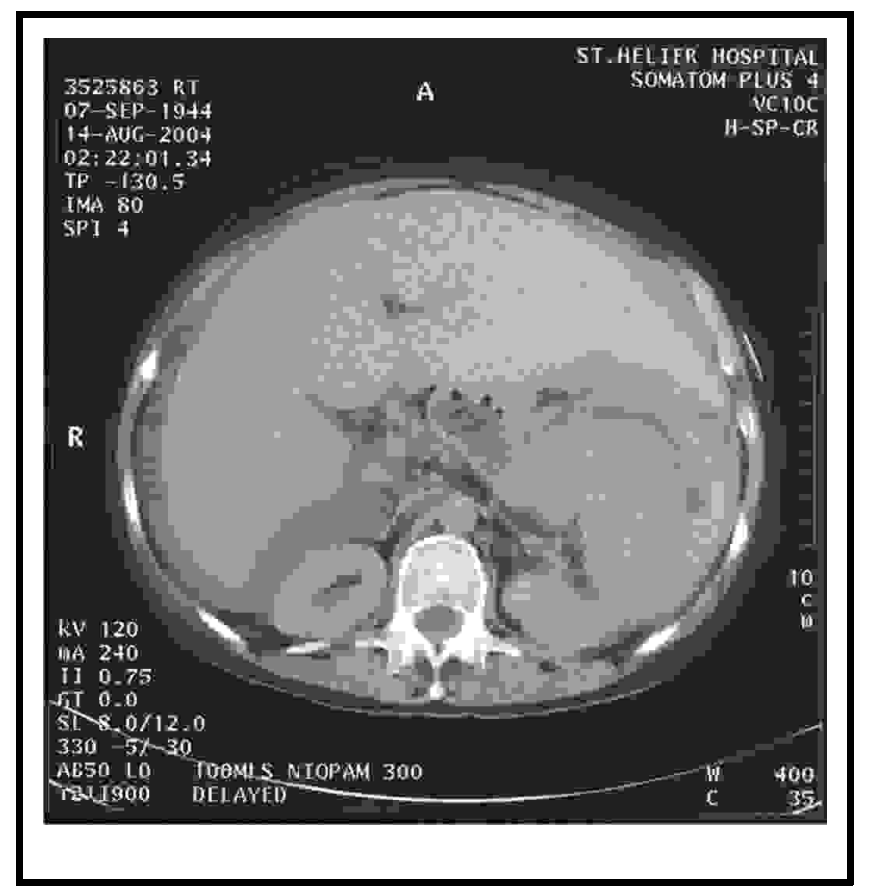

The spleen is involved mainly in AA and AL type of amyloidosis. There are two documented patterns of splenic amyloidosis a nodular form involving lymphoid follicles and a diffuse form infiltrating the red pulp. However splenomegaly is evident in only $4-13 \%$ of cases and splenic weight varies from 40 to $1400 \mathrm{~g}$ in autopsy studies. Hyposplenism is seen in around $25 \%$ of cases and is a poor prognostic factor as it indicates heavy deposition of amyloid in the spleen ${ }^{3}$.

Nakagawa studied the amyloid induced ultra structural changes in cell membrane by electron microscopy and found three types of amyloid deposition in capillary walls. 
In one form there were fine granular substances termed "pre amyloid" as well as amyloid fibrils deposited between basement membrane and endothelial cells of the capillary walls. In the second these deposits accumulated in the junctions between the endothelial cells and gradually increased the gap. In the third type called 'microcytosis indentation type', pinocytic vesicles form deep invaginations into the plasma membrane of the cell. All this deposition leads to widening of the gap between endothelial cells leading to vascular fragility. The platelet interaction with this amyloid deposition may also be defective and predispose spontaneous haemorrhage ${ }^{4}$.

Clotting factor deficiencies are also noted and Factor $X$ deficiency, which is the most common, is evident in 6.3$14 \%$ of cases. Experience from Boston Medical Centre suggests that $75 \%$ of patients with severe Factor $X$ deficiency had haemorrhagic complications of which $50 \%$ were severe and $17 \%$ were fatal ${ }^{5}$.

In our case the patient also recently received Autologous Stem Cell Transplant with Granulocyte colony stimulating factor (G-CSF) and high dose Melphalan treatment which results in reseeding of haematopoietic cells from bone marrow to the spleen resulting in increased splenic weight. This results in increased extramedullary haematopoiesis and splenic congestion, which can lead to capsular distension and tear ${ }^{5}$.
Thus in a case of occult haemorrhage with Amyloidosis, diagnosis of rupture of spleen should be considered and CT scan should be performed if the patient is haemodynamically stable. Splenectomy is the ideal treatment for ruptured spleen. Laparoscopic Splenectomy in acute situations is still in the experimental stages.

\section{REFERENCES}

1. Juchems MS, Aschoff AJ, Brambs HJ, Pauls S. Impact of a guiding CT prior to angiographic intervention. Rontgenpraxis. 2003;55(3):91-8.

2. D. Debnath and D. Valerio Atraumatic rupture of the spleen in adults. J.R.Coll.Surg.Edinb., 47, February 2002, 437-445

3. Kozicky OJ, Brandt LJ, Lederman M, Milcu M. Splenic amyloidosis: a case report of spontaneous splenic rupture with a review of the pertinent literature. Am J Gastroenterol. 1987 Jun; 82(6):582-7.

4. Nakagawa S. Ultrastructural aspects of cell membrane in amyloidogenesis and amyloid fibril destruction. In: GlennerGG, Costa PP, Freitas AF,eds. Amyloid and Amyloidogenesis. Proceedings of the third international symposium on amyloidosis. Amsterdam: Excerpta Medica 1980, S. 436-447.

5. Oran B, Wright DG, Seldin DC, McAneny D, Skinner M, Sanchorawala $V$. Spontaneous rupture of the spleen in AL amyloidosis. Am J Hematol. 2003 Oct; 74(2): 131-5. 